\section{Probing the olfactory code}

\author{
C. Giovanni Galizia and Randolf M enzel
}

\section{Multielectrode recording arrays in the moth antennal lobe indicate that the relative timing of action potentials may convey information about odor concentration and mixture.}

The olfactory system has a particularly difficult task: unlike vision or sound, the number of elementary stimuli- the odors- seems to be almost infinite. Furthermore, airborne odors come in plumes that reach the olfactory organ intermittently and unpredictably. A single plume may be very short, and successive plumes may come at a high frequency or after long intervals. The odor concentration in different plumes is highly variable and contains only scarce information about the odor source. To complicate things, behaviorally relevant odors are often blends of several substances, for which the composition and relative proportion of Odors must also be recognized against a background of confounding odors, which are always present in the natural environment, and context variables (such as time of day, developmental stage and motivational conditions) may change the importance and meaning of an odor. It is not surprising, then, that both neuroscientists and behavioral zoologists are greatly interested in how the brain processes olfactory information and ultimately identifies a relevant odor. However, this olfactory code is far from being understood. What weknow for sure is that many neurons are involved, and that to understand how understand the relationship between various levels of spatial and temporal information. In this issue, Christensen et al. ${ }^{1}$ take a big step forward by simultaneously measuring responses from several neurons in the olfactory system of a living insect.

The first brain structure to process olfactory information is the olfactory bulb in vertebrates, or its functional and structural analogue in insects, the antennal lobe (Fig. 1). The receptor cells that project to the antennal lobe come in different functional classes, which are

The authors are at the Institut für Biologie, NeurobiologieFreie Universität Berlin, KöniginLuise Str. 28-30, D-14195 Berlin, Germany. email: galizia@zedat.fu-berlin.deor menzel@neurobiologie.fu-berlin.de the blends' components are important. odor information is encoded, we have to defined by the complement of olfactory receptors that they express. In most cases, a receptor cell only expresses a single olfactory receptor gene; each class is selective for a number of chemical substances (odors), and the receptors respond in a dose-dependent manner. These properties define the cell's molecular receptive range, which may overlap for different receptor cells. Consequently, each odor elicits a pattern of activated receptor cell axons in the antennal lobe or olfactory bulb. In all cases studied so far, glomeruli receive inputs from a single or at most a few classes of receptor cell $s^{2-4}$. What is the nature of the output from these structures, and how are odor concentrations, specific blends and the temporal structure of the odor stimuli encoded?

Within the antennal lobe or olfactory bulb, the chemical identity of an odor (odor quality) is represented by patterns of active glomeruli and their output neurons, providing a spatial, combinatorial neural code $e^{5,6}$. The discovery of odorinduced oscillations in olfactory systems throughout the animal kingdom ${ }^{7}$ suggested that the identity of an odor may also be coded in the synchronous timing of action potentials in different neurons ${ }^{8}$. These oscillations arise from the activity of many neurons, and are driven by local neurons in the antennal lobe. Intracellular recordings from pairs of olfactory output neurons show stimuluscharacteristic patterns of synchronized activity, which are in tune with oscillations of a local field potential; single spikes ride on top of the oscillations. H owever, spiketiming could also codefor odor concentration or for a particular blend ${ }^{1}$, and the identity of the odor could be coded in the identity of the activated glomeruli ${ }^{9}$. To understand the relationship between the spatial and thetemporal properties of output neuron responses, we need simultaneous recordings from many neurons within the antennal lobe or olfactory bulb. The identity of the glomeruli that they innervate must be known, and different concentrations as well as odor mixtures need to betested.

In pioneering work, Christensen et al. ${ }^{1}$ now lay the foundations for such detailed studies. They took advantage of the extensively studied sexualpheromone system in the moth $M$ anduca sexta, and the technique of multiunit extracellular recording, which has been developed for the mammalian brain ${ }^{10,11}$. The application of multielectrode techniques to the insect brain and the olfactory system represents a new

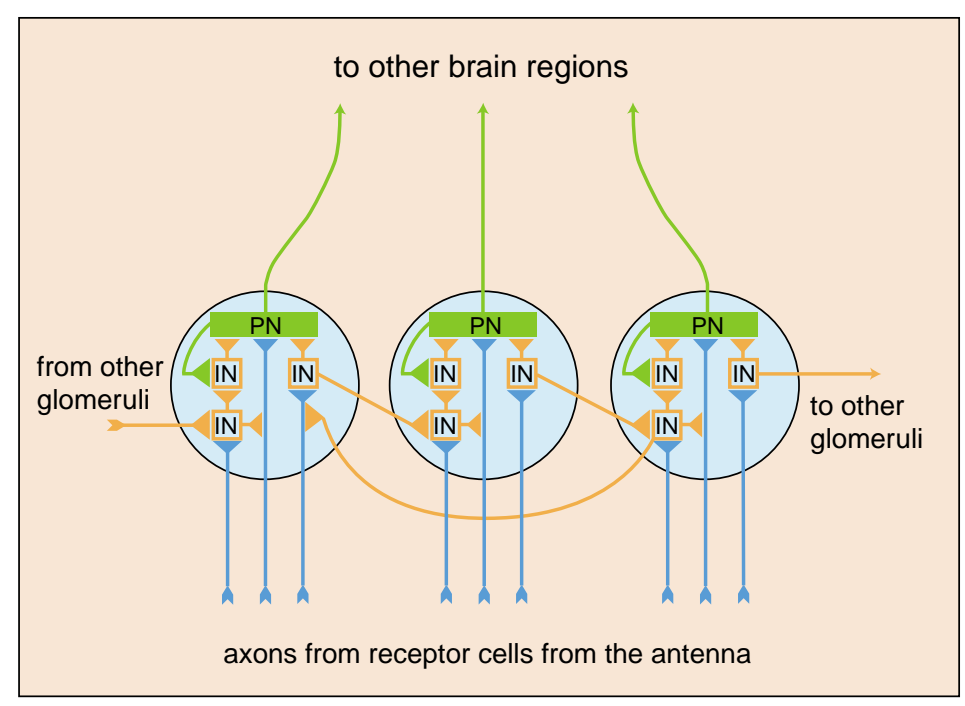

Fig. 1. A schematic diagram of the insect antennal lobe (analogous to the olfactory bulb in vertebrates). Axons from olfactory receptor cells enter the structure and branch within small balls of axons and dendrites called olfactory glomeruli, which line the outside of the antennal lobe. A selection of the numerous connections within and between the glomeruli are illustrated (IN , local interneurons). Projection neurons (PN, analogous to mitral/tufted cells in vertebrates) relay the information to higher brain areas. 


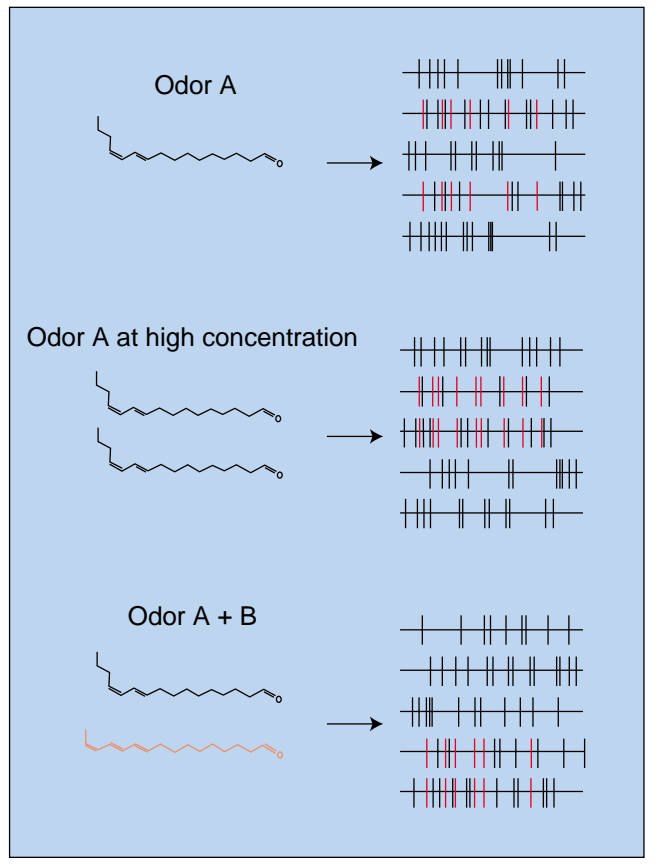

Fig. 2. The relative timing of action potentials may convey information about odor concentration and mixture. Christensen and colleagues presented a moth (Manduca sexta) with a pulse of pheromone (odor A, E10,Z12-hexadecadienal) at two different concentrations, as well as a mixture of two pheromones (odor $\mathrm{A}+$ odor $\mathrm{B}$, E10,E12,Z14-hexadecadienal), while recording simultaneously from multiple neurons in the antenna lobe (five traces shown here; data are schematic). Coincident spikes are labeled in red. N ote that in the three different conditions, different pairs of neurons share coincident spiking events.

and exciting development. Because the single electrodes in the array are $150 \mu \mathrm{m}$ apart, they record activity from different glomeruli, and thus permit analysis of the contribution of different glomeruli to the odor response. Furthermore, the electrode tip design allows for isolation of multiple neurons within a given glomerulus as well.

The study concentrated on the prominent macroglomerular complex (MGC) in the antennal lobe of the male moth, which is devoted to processing sexual pheromone information. The female moth attracts the male over long distances by releasing a pheromone blend, and the MGC contains highly specialized glomeruli that are selective for individual substances of the blend ${ }^{12,13}$. Christensen and colleagues ${ }^{1}$ now confirm that individual antennal lobe neurons have differing response profiles in that they responded differently to different odors and to the blend. By simultaneously recording up to seven neurons in each animal, the authors also analyzed the temporal coincidence of the action potentials recorded across the different electrodes, revealing two interesting novelties. First, no oscillatory responses were apparent. Second, synchronicity between units was not a function of the odor, but rather of the odor concentration and the specific blend (Fig. 2). Consequently, spatial and temporal patterns may encode different aspects of an olfactory stimulus. Odor quality may be encoded in the identity of the active output fibers (thus in the spatial component of the odor code), whereas stimulus properties such as concentration and blend composition could be encoded in the relative timing of output neuron action potentials. With the new approach reported here $^{1}$, a thorough investigation of these hypotheses seems to be within reach. Because of our considerable knowledge about the insect olfactory system, it will be possible to apply this method to neurons for which the innervated glomeruli are identified. Furthermore, multineuron recordings may be applied to animals performing learning tasks. Perhaps the electrodes could even be miniaturized and chronically implanted in a free-moving and behaving insect. This would lead the way to investigating the olfactory code under natural conditions.

Although some of the reported findings seem to contradict previous studies, these discrepancies are more likely a measure of our ignorance of the olfactory system. Christensen et al. did not find oscillations when they stimulated with short odor pulses ${ }^{1}$. In the sexual pheromone system, single odor plumes may be very short, because the continous filament is easily broken down into small plumes by air turbulences. In some species, females even release the pheromones as individual pulses. This would make it necessary for the olfactory system to identify an odor without the need for oscillatory activity. In the same system, however, oscillations appear with longer odor stimuli ${ }^{14}$. In these instances, oscillations may arise as a consequence of the wiring within the antennal lobe, because several recurrent excitatory and inhibitory connections easily form an oscillatory circuit, but may have no relevancefor the olfactory code.

The highly specialized pheromone sensitive macroglomerular complex, designed to optimize speed and sensitivity, may thus use different strategies than the 'ordinary' olfactory glomeruli, in encoding odors without the need for oscillations. This finding, therefore, does not contradict the hypothesis that oscillations may be useful in improving odor identification for general odors. Indeed, when the GABAergic neurons responsible for synchronization are pharmacologically blocked, honeybees lose their ability to distinguish between similar odors ${ }^{15}$. This exemplifies the idea that, despite the architectural similarity of different olfactory systems, results from one model cannot be directly extrapolated to other systems. The beauty of the new technique also lies in its suitability for investigating ordinary glomeruli as well as glomeruli devoted to processing sexual pheromones, in both insects and mammals alike. This will tremendously improve our understanding of glomerular organization. Multiunit recordings are now joining intracellular recordings and optical imaging in the quest to decipher the olfactory code.

1. Christensen, T. A., Pawlowski, V. M ., Lei, H. \& Hildebrand, J. G. Nat. Neurosci. 3, 927-931 (2000).

2. Hansson, B. S., Ljungberg, H., Hallberg, E. \& Löfstedt, C. Science 256, 1313-1315 (1992).

3. Mombaerts, P. Curr. Opin. Neurobiol. 6, 481-486 (1996)

4. Gao, Q., Yuan, B. \& Chess, A. Nat. Neurosci. 3, 780-785 (2000).

5. Galizia, C. G., Sachse, S., Rappert, A. \& Menzel, R. Nat. Neurosci. 2, 473-478 (1999).

6. Mori, K., Nagao, H. \& Yoshihara, Y. Science 286, 711-715 (1999).

7. Gelperin, A. J. Exp. Biol. 202, 1855-1864 (1999).

8. Laurent, G. Curr. O pin. Neurobiol. 7, 547-553 (1997).

9. Laurent, G. Science 286, 723-728 (1999).

10. Nicolelis, M. A., Ghazanfar, A. A., Faggin, B. M., Votaw, S. \& Oliveira, L. M. Neuron 18, 529-537 (1997).

11. Sutherland, G. R. \& McNaughton, B. Curr. Opin. Neurobiol. 10, 180-186 (2000).

12. Hansson, B. S. \& Christensen, T. A. in Insect Olfaction (ed. Hansson, B. S.) 125-161 (Springer, Berlin, 1999).

13. Heinbockel, T., Christensen, T. A. \& Hildebrand, J. G. J. Comp. Neurol. 409, 1-12 (1999).

14. Heinbockel, T., Kloppenburg, P. \& Hildebrand, J. G. J. Comp. Physiol. A 182, 703-714 (1998)

15. Stopfer, M., Bhagavan, S., Smith, B. H. \& Laurent, G. Nature 390, 70-74 (1997). 